Commun. Theor. Phys. 66 (2016) 269-274 Vol. 66, No. 3, September 1, 2016

\title{
Entropy, Fisher Information and Variance with Frost-Musulin Potenial
}

\author{
J.O.A. Idiodi and C.A. Onate* \\ Physics Department, University of Benin, Benin, Nigeria
}

(Received February 22, 2016; revised manuscript received April 19, 2016)

\begin{abstract}
This study presents the Shannon and Renyi information entropy for both position and momentum space and the Fisher information for the position-dependent mass Schrodinger equation with the Frost-Musulin potential. The analysis of the quantum mechanical probability has been obtained via the Fisher information. The variance information of this potential is equally computed. This controls both the chemical properties and physical properties of some of the molecular systems. We have observed the behaviour of the Shannon entropy. Renyi entropy, Fisher information and variance with the quantum number $n$ respectively.
\end{abstract}

PACS numbers: 03.65.Ca, 03.65.Ta, 89.70.+c, 31.15.Ew, 02.50.Cw

Key words: Fisher information, Shannon entropy, position-dependent mass

Available at: www.iopscience.iop.org. 